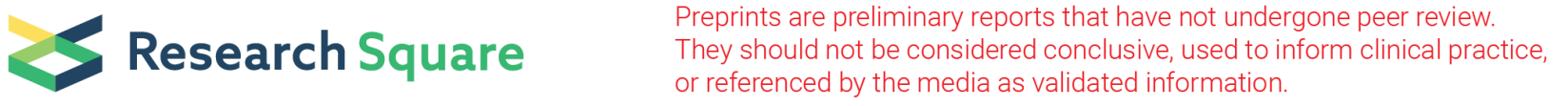 \\ Altered regional gray matter volume in Chinese female patients with bulimia nervosa
}

\section{Xiao Li}

Sichuan University https://orcid.org/0000-0002-4233-3378

\section{Xiaowei Liu}

Sichuan University West China Hospital

\section{Yu Wang}

Sichuan University West China Hospital

\section{Lingfei Li}

Sichuan University West China Hospital

\section{Linli Zheng}

Sichuan University West China Hospital

\section{Yaya Liu}

Zunyi psychiatric hospital, Guizhou province

\section{Jing Ma}

Sichuan University West China Hospital

\section{Lan Zhang ( $\nabla$ huaxizhanglan@126.com ) \\ Sichuan University West China Hospital}

\section{Research article}

Keywords: bulimia nervosa, MRI, grey matter volume, voxel-based morphometry

Posted Date: February 4th, 2020

DOI: https://doi.org/10.21203/rs.2.17263/v3

License: @) (1) This work is licensed under a Creative Commons Attribution 4.0 International License. Read Full License

Version of Record: A version of this preprint was published at BMC Psychiatry on March 2nd, 2020. See the published version at https://doi.org/10.1186/s12888-020-02493-4. 


\section{Abstract}

Background The bulimia nervosa (BN) is one of the psychiatric disorders, which pathophysiology is not clear currently. Recent studies have concentrated on brain structural and functional changes in BN patients. However, findings have been inconsistent. The aim of this study was to explore the relationships between brain structural alterations and clinical features in Chinese female patients with BN.

Method A total of 34 female patients with BN and 34 matched healthy controls ( $\mathrm{HC}$ ) underwent a T1-weighted magnetic resonance imaging (MRI) scanning. VBM analysis was carried out by using SPM8 ( http://www.fil.ion.ucl.ac.uk/spm/software/spm8/ ) to explore the alterations of regional Grey Matter Volume (GMV). The correlation analysis was performed using Statistical Package for the Social Sciences (SPSS) .

Results The BN group showed reduced regional grey matter volume of the left medial superior frontal gyrus (SFGmed.L), the right superior temporal gyrus (STG.R), the right median cingulate and paracingulate gyri (DCG.R), the left median cingulate and paracingulate gyri (DCG.L) and the left dorsolateral superior frontal gyrus (SFGdor.L) compared to HC. There was no significantly regional grey matter volume increase in BN patients. The brain areas that GMV altered were uncorrelated with $\mathrm{BMI}$ and duration of illness ( $p>0.05)$. There was a significantly negtive correlation between age and GMV in the SFGmed.L $(P<0.005, r=-0.516)$, the DCG.R $(P<0.005, r=-0.556)$, the DCG.L $(P<0.05, r=-0.576)$ and the SFGdor.L $(P<0.005, r=-0.576)$. The self-esteem and self-evaluation of BN patients were uncorrelated with the structural alterations of GMV.

Conclusions The results show altered regional GMV in females with bulimia nervosa in several brain areas, but it difficult to differentiate whether the GMV changes are the results of BN pathology or the consequences of binge-eating and compensatory behaviour. The structural alterations might be associated with impairments of inhibitory control and body dissatisfaction in BN patients, as well as mood disorder comorbidity.

\section{Background}

Bulimia nervosa (BN) is an eating disorder characterized by recurrent episodes of binge eating and inappropriate compensatory behaviors to prevent weight gain [1]. It usually occurs in young females and can lead to severe medical complications affecting all body systems $[2,3]$. The neurobiological processes underlying BN are unclear. Several studies have provided evidence that BN and other eating disorders are associated with structural and functional changes in brain regions, as detected using magnetic resonance imaging (MRI) (Table 1). For example, studies based on voxel-based morphometry (VBM) from MRI images have associated BN with increased grey matter volume (GMV) in the medial orbitalfrontal cortex and the ventral striatum[4], as well as in the insula [5], lingual gyri and inferior parietal lobule [6]. These and other studies also found reduced GMV in caudate and putamen [5, 7], bilateral medial frontal, precentral gyri, right postcentral gyrus, left superior (SFG) and inferior frontal gyri [6]. Some brain structural studies have found cortical abnormalities in BN, such as reduced cortical thickness in the bilateral frontal, temporal and parietal lobes, negtively related to the severity of BN symptoms[8]; and reduced cortical thickness in the right pars triangularis, right superior parietal and left dorsal posterior cingulate cortices as well as greater cortical thickness in the left ventral posterior cingulate cortex [9]. These and other brain structural studies have found abnormal structural connectivity in BN. Greater structural connectivity has been found among the insula, orbitofrontal cortex and ventral striatum, as well as among the bilateral frontal, temporal and parietal lobes. Conversely, lower connectivity has been found in the orbitofrontal cortex, amygdala and hypothalamus $[9,10]$.

These various studies have given inconsistent findings, likely reflecting small samples, differences in patient populations, and differences in methodology. For example, some studies corrected for age and total intracranial volume (TIV), while others corrected for age and body mass index (BMI). Several studies did not consider potential effects of medication. Many studies have not examined whether structural changes in the brain correlate with low self-esteem and self-evaluation, which are core features of BN and other eating disorders $[11,12]$. 
The present study aimed to help clarify the potential association of BN with altered GMV in the brain, and to examine whether these structural changes correlate with patient's self-evaluation. We retrospectively examined a small population of Chinese women with $\mathrm{BN}$ at our medical center, comparing them to a matched group of healthy controls.

\section{Methods}

\subsection{Participants}

This retrospective study involved 34 women diagnosed with BN at the Mental Health Center of West China Hospital, Sichuan University. The patients had engaged in binge eating and compensatory behaviors at least once a week during the three months prior to enrollment in the study. As controls, we recruited 34 age-matched women with BMI within the normal range of 18.5-23.9 who reported no history of eating disorders or other psychiatric disorders. Controls were recruited from the community through public advertisements. This study was approved by the Ethics Committee of West China Hospital, and all subjects provided written informed consent.

Patients and controls were assessed by two psychiatrists using the structured clinical interview for DSM-V disorders (SCID). All study subjects were right-handed, and none was currently taking medications or reported a history of major psychiatric disorders, head trauma, substance abuse or dependence, or neurologic disease. None of the subjects reported having metal implants or a pacemaker. Nine BN patients had mild anxiety and depressive symptoms based on the SCID, but we did not exclude them because these symptoms occur often in BN $[13,14]$.

\subsection{Clinical psychological assessment of patients}

All patients completed the revised Chinese version of the Core Self-Evaluation Scale (CSES) and the Chinese version of the Rosenberg Self-Esteem Scale (RSES). The revised Chinese CSES uses 10 items to assess core self-evaluation, and it shows acceptable reliability and validity $[13,14]$. The survey applies a 5-point scoring method, and the total score ranges from 10 to 50 points. The national norm on the CSES is $36.05 \pm 5.21$ for Chinese undergraduate women [15], and CSES scores of our patients were compared to this norm.

The RSES assesses self-esteem [16] and includes 10 items, with items 3, 5, 8, 9, 10 negatively worded. Each item is scored on a 4-point scale, and the individual item scores are summed to obtain a total score. We compared the RSES scores of our patients to the mean RSES score of $28.73 \pm 4.48$ reported for undergraduates in Beijing [17]. This norm did not vary significantly with gender in that study.

\subsection{MRI data acquisition}

MR images were obtained using a Philips 3.0 T system. All subjects underwent 3D T1-weighted volumetric scanning with the following parameters: orientation, sagittal; matrix size, 256×256; field of view (FOV), $256 \times 256 \mathrm{~mm}$; slice thickness, $1 \mathrm{~mm}$; gap, none; flip angle, $7^{\circ}$; repetition time (TR), $8.2 \mathrm{~ms}$; echo time (TE), $3.8 \mathrm{~ms}$; voxel size, $1 \times 1 \times 1 \mathrm{~mm}$. During MRI, all subjects were asked to relax, keep their eyes closed without falling sleep and their head as motionless as possible, and not to think of anything in particular.

\subsection{VBM}

MRI data were analyzed using SPM8 (Wellcome Trust Centre for Neuroimaging, Institute of Neurology, London, UK) and the VBM toolbox VBM8 (www.neuro.uni-jena.de/vbm/), both run within Matlab R2013b. Structural images were normalized to 
Montreal Neurological Institute $\triangle \mathrm{MNI} \square$ standard space and segmented into grey matter (GM), white matter (WM) and cerebrospinal fluid (CSF) using the unified segmentation approach. Finally, grey matter images were smoothed using an 8mm full-width half-maximum Gaussian kernel.

\subsection{Statistical analysis}

GMV in different brain regions was compared between patients and controls using the two-samples $t$ test in SPM8. Covariates were age and TIV, defined as the sum of GM, WM and CSF volumes. Multiple comparisons were corrected using the familywise error approach at a cluster level, with a corrected threshold of $\mathrm{P}<0.001$. An absolute threshold was set at 0.2 in all computations.

The two-samples $t$ test in SPSS 20.0 (IBM, Chicago, IL, USA) was used to compare patients and controls in terms of clinicodemographic data as well as total GM, WM, CSF and intracranial volumes. Regions of interest (ROIs) showing GMV changes were defined using the "region of interest extraction" tool in xjView (www.alivelearn.net/xjview8/) via the SPM toolbox. Pearson correlation analysis was conducted within SPSS to explore relationships between ROls and clinical variables after Bonferroni correction, with significance defined as $\mathrm{P}<0.01$.

\section{Results}

3.1 Clinico-demographics characteristics of participants

Table 2 summarizes the demographics and clinical characteristics of patients and healthy controls. The groups did not differ significantly in age, $\mathrm{BMI}$ or years of education $(P>0.05)$. Patients showed lower mean RSES and CSES scores than the corresponding norms from healthy populations (see Methods).

\subsection{VBM}

\subsubsection{Whole brain tissue volumes}

There were no significant differences between patients and controls in total GMV, WMV or TIV $(P>0.05$, Table 3). In contrast, patients showed a larger total CSF volume.

\subsubsection{Regional GMV}

Using age and TIV as confounding covariates in VBM analysis, we identified five clusters of regional GMV after correction for multiple comparisons at the cluster level (threshold $P<0.001$ ). Patients showed lower GMV in the left medial superior frontal gyrus (SFGmed.L), right superior temporal gyrus (STG.R), right median cingulate and paracingulate gyri (DCG.R), left median cingulate and paracingulate gyri (DCG.L) and left dorsolateral superior frontal gyrus (SFGdor.L) (Table 3 and Figure 1). In none of the regions did patients show increased GMV.

\subsection{Correlations of GMV reduction with patient characteristics}

$\mathrm{BN}$-associated GMV reductions did not vary significantly with BMI, duration of illness, or total scores on the RSES or CSES (P $>0.05)$. In contrast, age did correlate negatively with GMV in the SFGmed.L $(r=-0.516, P<0.005)$, DCG.R $(r=-0.556, P<$ 0.005), DCG.L $(r=-0.576, P<0.05)$ and SFGdor.L $(r=-0.576, P<0.005)$ (Figure 2$)$. 


\section{Discussion}

\subsection{Global brain volume alterations}

We found no significant differences between BN patients or controls in total GMV, WMV or intracranial volume, which is consistent with some previous studies in female patients[18, 19]. Our patients did show increased CSF volume, consistent with results obtained with one group of German patients [20] but not with a second German group [18]. These differences may reflect differences in methodology. Our observation of increased CSF volume but unchanged TIV suggests that the reduced GMV in BN is replaced by increased CSF.

\subsection{Regional GMV alterations}

Patients showed reduced GMV in the SFGmed.L, STG.R, DCG.L, DCG.R and SFGdor.L, and no regions with increased GMV. These results are consistent with some studies but not with others, which have reported reduced GMV in the superior frontal gyrus and cingulate cortices [6], increased GMV in the medial orbital frontal cortex and the ventral striatum [4], or increased GMV in the paracentral lobule, precuneus, left putamen and insula, but reduced GMV in the caudate nucleus and thalamus [19]. Thus, different studies of BN patients vary considerably in the regions showing GMV alterations and the direction of the alteration (increase or decrease). These discrepancies may be due to differences in disease course, disease stage, medication history, ethnicity and other factors. Future studies should strive to examine larger, multi-center populations that may help reduce the influence of clinico-demographic factors on VBM.

The SFG is located in the superior part of the prefrontal cortex, which plays essential roles in executive control [21], including self-regulation of eating behavior [22]. The structural and functional abnormalities in the SFG in patients with BN or other eating disorders have been associated with deficits in self-regulation and reward processing [23]. Functional MRI studies have shown the SFG to be deactivated in BN in the presence of the expected reward during a reward-based learning task [24], during a Simon spatial incompatibility task [23]. The prefontal cortex is an important part of the fronto-striatal circuits, which is involved in self-regulatory control. Previous study has found deficient activity in the fronto-striatal circuits associated with impaired self-regulatory processes $[23,25]$. The prefrontal cortex is also an important component of the forebrain system, which may contribute to eating dysregulation by driving maladaptive over- and undereating [26]. These alterations are likely lead to binge eating behaviors, thereby contributing to the development and maintenance of BN.

Reduced STG volume may cause abnormal body image perception and excessive concern about body shape and weight, leading to restrictive or binge eating. The GMV reduction in the STG in the Japanese study was associated with body dissatisfaction [27]. A functional MRI study has shown deactivation in STG when the individual was thinking about eating food [28]. Patients with eating disorders such as BN typically show emotional symptoms such as depressive and anxiety disorders $[14,29]$. How these symptoms relate to inappropriate eating behaviors is controversial. Many studies have suggested that inappropriate eating behaviors are maladaptive strategies to manage negative feelings [30]. However, other studies have suggested that the eating behaviors alter mental state, such as by activating serotonin projections of the dorsal raphe to the prefrontal cortex; as a result, normalizing eating behavior can normalize psychiatric symptoms [31]. We found reduced GMV volume in the STG of our BN patients, which may link to more severe symptoms of depression and anxiety disorders [32,33]. Such a reduction has also been linked to risk of suicide attempts in community samples of adolescents [34]. Further studies should examine whether such GMV reductions precede or follow the onset of inappropriate eating behaviors.

We observed reduced GMV in the middle cingulate cortex, and such a reduction has been rarely reported in BN. The middle cingulate cortex is associated with inhibitory functions and self-control, GMV in the middle cingulate cortex negtively correlated with uncontrlled eating behaviors has been reported in normal-weight female undergraduate students [35]. In 
addition, obese individuals in one study showed reduced activation of the middle cingulate cortex during appetite control [36]. The structural and functional alterations of the middle cingulate cortex in BN patients should be explored further.

\subsection{Correlations between GMV alterations and clinical variables}

BN-associated GMV reductions did not vary significantly with BMI, duration of illness, or total scores on the RSES or CSES. Age did, however, correlate negatively with GMV in the SFGmed.L, DCG.R, DCG.L and SFGdor.L.

As expected, RSES and CSES scores were lower in BN patients than in controls, reflecting that a frequent symptom of BN is body image dissatisfaction [37], which is linked to low self-esteem [38, 39]. At the beginning of this study, we hypothesized that the RSES and CSES scores of BN patients would be associated with GMV alterations. However, we did not observe such an association in our patients. In contrast, a study of BN patients in the US did detect an association between brain functional alterations and self-esteem: activation of the right temporoparietal junction, precuneus and dorsal anterior cingulate cortex was weaker in BN patients than in controls during execution of social and self-knowledge tasks [40]. These brain regions are associated with self-knowledge and social processing. The discrepancy between that study and ours may be due to methodological differences. Whether the GMV reductions in our BN patients correlate with functional changes affecting selfesteem and self-evaluation should be explored further.

\subsection{Study limitations}

This study analyzed only women, even though some men are also affected by eating disorders. In addition, some of our patients had mild anxiety and depressive symptoms, which may have confounded our results but which may also make our sample more representative of typical BN patient populations [14]. The cross-sectional nature of our study means that further work is needed to clarify whether the observed GMV alterations are the result of BN pathology or the consequence of bingeeating and compensatory behaviors. Our results should be verified and extended with much larger samples. In our study, we explored only brain structural alterations in BN; future work should explore changes in brain functional connectivity and networks in order to understand how brain areas interact with one another.

\section{Conclusions}

The present study showed regional GMV decreases in several brain areas of women with BN. These structural alterations may be associated with impaired inhibitory control, body dissatisfaction, and emotion dysrugulation. While these GMV reductions correlated with patient age, they did not correlate with self-esteem or self-evaluation. These findings should be verified and extended in larger, preferably longitudinal studies.

\section{Abbreviations}

AN: anorexia nervosa

BMl: body mass index

BN: bulimia nervosa

CSES: Core Self-Evaluation Scale

CSF: cerebrospinal fluid

DCG.L: left median cingulate and paracingulate gyri 
DCG.R: right median cingulate and paracingulate gyri

GM: grey matter

GMV: grey matter volume

MRI: magnetic resonance imaging

ROI: region of interest

RSES: Rosenberg Self-Esteem Scale

SCID: Structured clinical interview for DSM-V disorders

SFGdor.L: left dorsolateral superior frontal gyrus

SFGmed.L: left medial superior frontal gyrus

SPSS: Statistical Package for the Social Sciences

STG.R: right superior temporal gyrus

TIV: total intracranial volume

TE: echo time

TR: repetition time

VBM: voxel-based morphometry

WMIwhite matter

WMV: white matter volume

\section{Declarations}

\section{Acknowledgments}

The authors would like to thank all subjects for contributing their time and efforts to this study.

\section{Funding}

This study was supported by funds for postgraduate training from Sichuan University.

\section{Authors' information}

XL, LFL, LLZ and JM are master's candidates at the Mental Health Center, West China Hospital of Sichuan University. XXL is a psychiatry resident and YW a PhD candidate at the Mental Health Center. YYL is a psychiatry resident at Zunyi Psychiatric Hospital in Guizhou province. 
Authors' contributions

LZ, YW and XL designed the study. LLZ, LFL, YYL and JM recruited patients and collected demographic data. XL, XWL and YW collected MRI data. XL and XWL analyzed and interpreted the results. XL drafted the manuscript, which $L Z$ revised. All authors read and approved the final manuscript.

\section{Corresponding author}

Correspondence to Lan Zhang.

\section{Ethics approval and consent to participate}

The study was approved by the Ethics Committee of West China Hospital of Sichuan University. All participants provided written informed consent.

\section{Consent for publication}

Not applicable.

\section{Availability of data and materials}

The datasets analyzed during the current study are available from the corresponding author on reasonable request.

\section{Competing interests}

The authors declare that they have no competing interests.

\section{References}

1. American Psychiatric Association: Diagnostic and statistical manual of mental disorder. 2013, Arlington VA: American Psychiatric Publishing, 5.

2. Gibson D, Workman C, Mehler PS: Medical Complications of Anorexia Nervosa and Bulimia Nervosa. PSYCHIAT CLIN N AM 2019, 42(2):263-274.

3. Sachs K, Mehler PS: Medical complications of bulimia nervosa and their treatments. Eating and Weight Disorders - Studies on Anorexia, Bulimia and Obesity 2016, 21(1):13-18.

4. Schäfer $A$, Vaitl $D$, Schienle A: Regional grey matter volume abnormalities in bulimia nervosa and bingeeating disorder. NEUROIMAGE 2010, 50(2):639-643.

5. Frank GK, Shott ME, Hagman JO, Mittal VA: Alterations in Brain Structures Related to Taste Reward Circuitry in III and Recovered Anorexia Nervosa and in Bulimia Nervosa. AM JPSYCHIAT 2013, 170(10):1152-1160.

6. Marsh R, Stefan M, Bansal R, Hao X, Walsh BT, Peterson BS: Anatomical Characteristics of the Cerebral Surface in Bulimia Nervosa. BIOL PSYCHIAT 2015, 77(7):616-623. 
7. Coutinho J, Ramos AF, Maia L, Castro L, Conceição E, Geliebter A, Machado PPP, Gonçalves Ó, Sampaio A: Volumetric alterations in the nucleus accumbens and caudate nucleus in bulimia nervosa: A structural magnetic resonance imaging study. INT J EAT DISORDER 2015, 48(2):206-214.

8. Westwater ML, Seidlitz J, Diederen KMJ, Fischer S, Thompson JC: Associations between cortical thickness, structural connectivity and severity of dimensional bulimia nervosa symptomatology. Psychiatry Research: Neuroimaging 2018, 271:118-125.

9. Berner LA, Stefan M, Lee S, Wang Z, Terranova K, Attia E, Marsh R: Altered cortical thickness and attentional deficits in adolescent girls and women with bulimia nervosa. J PSYCHIATR NEUROSCI 2018, 43(3):151-160.

10. Frank GKW, Shott ME, Riederer J, Pryor TL: Altered structural and effective connectivity in anorexia and bulimia nervosa in circuits that regulate energy and reward homeostasis. TRANSL PSYCHIAT 2016, 6(11):e932.

11. Boucher, K; Côté, M; Gagnon-Girouard, MP; Bégin, C: Eating Pathology Among Patients With Anorexia Nervosa and Bulimia Nervosa: The Role of Narcissism and Self-Esteem. J. Nerv. Ment. Dis 2018, 206: 776-782.

12. Blechert J, Ansorge U, Beckmann S, Tuschen-Caffier B: The undue influence of shape and weight on self-evaluation in anorexia nervosa, bulimia nervosa and restrained eaters: a combined ERP and behavioral study. PSYCHOL MED 2011, 41(1):185-194.

13. Hudson Jl, Hiripi E, Pope HG, Kessler RC: The Prevalence and Correlates of Eating Disorders in the National Comorbidity Survey Replication. BIOL PSYCHIAT 2007, 61(3):348-358.

14. Godart N, Radon L, Curt F, Duclos J, Perdereau F, Lang F, Venisse JL, Halfon O, Bizouard P, Loas G et al: Mood disorders in eating disorder patients: Prevalence and chronology of ONSET. J AFFECT DISORDERS 2015, 185:115-122.

15. DuJianzheng ZZ: Reliability, validation and construct confirmatory of core self-evaluation. Psychological Research 2012(03):54-60.

16. Rosenberg: Society and the adolescent self-image. United States of America: Princeton University Press; 1965.

17. Hong L: Research on the self-esteem of college students. Studies of Psychology and Behavior 2003(02):133-136.

18. Joos A, Klöppel S, Hartmann A, Glauche V, Tüscher O, Perlov E, Saum B, Freyer T, Zeeck A, van Elst LT: Voxel-based morphometry in eating disorders: Correlation of psychopathology with grey matter volume. Psychiatry Research: Neuroimaging 2010, 182(2):146-151.

19. Amianto F, Caroppo P, D Agata F, Spalatro A, Lavagnino L, Caglio M, Righi D, Bergui M, Abbate-Daga G, Rigardetto R et al: Brain volumetric abnormalities in patients with anorexia and bulimia nervosa: A Voxel-based morphometry study. Psychiatry Research: Neuroimaging 2013, 213(3):210-216.

20. Krieg JC, Lauer C, Pirke KM: Structural brain abnormalities in patients with bulimia nervosa. Psychiatry Res 1989, 27(1):39-48.

21. Funahashi S, Andreau JM: Prefrontal cortex and neural mechanisms of executive function. Journal of Physiology-Paris 2013, 107(6):471-482.

22. Dohle, S; Diel, K; Hofmann, W: Executive functions and the self-regulation of eating behavior: A review. Appetite 2018, 124: 4-9.

23. R M, G H, Z W, P W, KW K, LA B, BT W, BS P: An FMRI study of self-regulatory control and conflict resolution in adolescents with bulimia nervosa. The American journal of psychiatry 2011, 168(11):1210-1220. 
24. M C, Z W, GZ T, G Z, E F, M S, K T, R M: Reward-Based Spatial Learning in Teens With Bulimia Nervosa. J AM ACAD CHILD PSY 2016, 55(11):962-971.

25. Marsh R, Steinglass JE, Gerber AJ, Graziano O Leary K, Wang Z, Murphy D, Walsh BT, Peterson BS: Deficient Activity in the Neural Systems That Mediate Self-regulatory Control in Bulimia Nervosa. Archives of General Psychiatry 2009, 66(1):51-63.

26. Petrovich GD: Forebrain networks and the control of feeding by environmental learned cues. PHYSIOL BEHAV2013, 121:10-18.

27. Kohmura K, Adachi Y, Tanaka S, Katayama H, Imaeda M, Kawano N, Nishioka K, Ando M, lidaka T, Ozaki N: Regional decrease in gray matter volume is related to body dissatisfaction in anorexia nervosa. Psychiatry Research: Neuroimaging 2017, 267:51-58.

28. SJ B, OG O, R U, HC F, V G, M B, SC W, HB S, J T, IC C: Differential neural responses to food images in women with bulimia versus anorexia nervosa. PLOS ONE 2011, 6(7):e22259.

29. Kaye WH, Bulik CM, Thornton L, Barbarich N, Masters K, The PFCG: Comorbidity of Anxiety Disorders With Anorexia and Bulimia Nervosa. AM J PSYCHIAT 2004, 161(12):2215-2221.

30. JM L, SA W, CB P, RD C, SG E, JE M, SJ C, TL S, MH K, AB G et al: Dimensions of emotion dysregulation in bulimia nervosa. European eating disorders review : the journal of the Eating Disorders Association 2014, 22(3):212-216.

31. loakimidis I, Zandian M, Ulbl F, Bergh C, Leon M, Södersten P: How eating affects mood. PHYSIOL BEHAV2011, 103(34):290-294.

32. van Tol MJ, van der Wee NJ, van den Heuvel OA, Nielen MM, Demenescu LR, Aleman A, Renken R, van Buchem MA, Zitman FG, Veltman DJ: Regional brain volume in depression and anxiety disorders. Arch Gen Psychiatry 2010, 67(10):10021011.

33. Lai C, Wu Y: The gray matter alterations in major depressive disorder and panic disorder: Putative differences in the pathogenesis. J AFFECT DISORDERS 2015, 186:1-6.

34. Pan LA, Ramos L, Segreti A, Brent DA, Phillips ML: Right superior temporal gyrus volume in adolescents with a history of suicide attempt. BRIT J PSYCHIAT 2015, 206(4):339-340.

35. Song S, Zhang Y, Qiu J, Chen H et al: Brain structures associated with eating behaviors in normal-weight young females. Neuropsychologia 2019, 133: 107171.

36. Tuulari JJ, Karlsson HK, Hirvonen J et al: Neural circuits for cognitive appetite control in healthy and obese individuals: an fMRI study. PLOS ONE 2015, 10(2): e0116640.

37. Bowden PK, Touyz SW, Rodriguez PJ, Hensley R, Beumont PJ: Distorting patient or distorting instrument? Body shape disturbance in patients with anorexia nervosa and bulimia. The British Journal of Psychiatry 1989, 155(2):196-201.

38. Meijboom A, Jansen A, Kampman M, Schouten E: An experimental test of the relationship between self-esteem and concern about body shape and weight in restrained eaters. Int J Eat Disord 1999, 25(3):327-334.

39. Laporta-Herrero I, Jáuregui-Lobera I, Barajas-Iglesias B, Santed-Germán MÁ: Body dissatisfaction in adolescents with eating disorders. Eating and Weight Disorders - Studies on Anorexia, Bulimia and Obesity 2018, 23(3):339-347.

40. McAdams CJ, Krawczyk DC: Neural Responses during Social and Self-Knowledge Tasks in Bulimia Nervosa. FRONT PSYCHIATRY 2013, 4. 


\section{Tables}

Page 11/16 
Table 1: A review table of the existing structural work in bulimia nervosa

$\overline{\text { Regional Differences }} \quad$ Total Volume

Difference

\begin{tabular}{|c|c|c|c|c|}
\hline & $\begin{array}{ll}\mathrm{BN}(\mathrm{n}=14) & \mathrm{BN}: 23.1(3.8)\end{array}$ & VBM & Increased GMV of the & Not assessed \\
\hline $\begin{array}{l}\text { Neuroimage.2010;50(2):639- } \\
43\end{array}$ & $\begin{array}{ll}\mathrm{BED}(\mathrm{n}=1 & \mathrm{BED}: 26.4(\mathrm{~b} .4) \\
\mathrm{HC}(\mathrm{n}=19) & \mathrm{HC}: 22.3(2.6)\end{array}$ & & $\begin{array}{l}\text { ventral striatum in } \\
\mathrm{BN}(\mathrm{BN}>\mathrm{HC}) \text {. }\end{array}$ & \\
\hline
\end{tabular}

Frank et.al(2013)

Am J

$\mathrm{AN}(\mathrm{n}=19)$

Recovered

Psychiatry.2013;170(10):1152-AN(n=24)

60

$\mathrm{BN}(\mathrm{n}=20)$

$\mathrm{HC}(\mathrm{n}=24)$

$\mathrm{BN}(\mathrm{n}=21)$

$\mathrm{HC}(\mathrm{n}=20)$

Int J Eat

Disord.2015;48(2):206-14

In this research

$\mathrm{BN}(\mathrm{n}=34)$

$\mathrm{HC}(\mathrm{n}=34)$
$\mathrm{BN}: 22.85(3.89)$

HC:22.26(2.53)

AN:23.1(5.8)

Recovered

AN:30.3(8.1)

$\mathrm{BN}: 25.2(5.3)$

$\mathrm{HC}: 27.4(6.3)$

$\mathrm{BN}: 31.57(8.27)$

HC:30.90(8.79)

VBM

VBM

Reduced GMV in the

SFGmed.L, STG.R,

DCG.R, DCG.L and

SFGdor.L $(\mathrm{BN}<\mathrm{HC})$.
$\mathrm{BN}(\mathrm{n}=34)$ $\mathrm{HC}(\mathrm{n}=34)$
Marsh et.al(2015)

Biol

Psychiatry.2015;77(7):616-23 $\mathrm{BN}: 21.6(6.0)$
$\mathrm{HC}: 22.08(6.5)$

Morphological Increased GMV in analysis including VBM and Cortical Thickness

$\mathrm{AN}(\mathrm{n}=26)$ $\mathrm{BN}(\mathrm{n}=25)$

Transl

Psychiatry.2016;6(11):e932
$\mathrm{AN}: 23.23(5.26)$ Structural

$\mathrm{BN}: 24.64(4.22)$ and effective HC:24.39(3.49) connectivity and bilateral temporoparietal areas $(\mathrm{BN}<\mathrm{HC})$.
Berner et al(2018)

J Psychiatry

Neurosci.2018;43(3):151-160
$\mathrm{BN}(\mathrm{n}=28) \quad \mathrm{BN}: 18.8(4.1) \quad$ Cortical $\mathrm{HC}(\mathrm{n}=21) \quad \mathrm{HC}: 19.2(5.5) \quad$ thickness lingual gyri and inferior not differ parietal lobule in across the BN $\mathrm{BN}(\mathrm{BN}>\mathrm{HC})$; reduced and control GMV in bilateral medial group $(P>0.05)$. frontal and precentral gyri, left superior and inferior frontal gyri, the right postcentral gyrus

Greater structural Not assessed connectivity in insula, orbitofrontal cortex and ventral striatum(BN>HC), but lower connectivity in orbitofrontal cortex, amygdala and hypothalamus in $\mathrm{BN}(\mathrm{BN}<\mathrm{HC})$.

Greater cortical thickness in the left ventral posterior cingulate cortex in $\mathrm{BN}(\mathrm{BN}>\mathrm{HC})$; reduced thickness of the right pars triangularis, right superior parietal and left dorsal posterior cingulate cortices $(\mathrm{BN}<\mathrm{HC})$.

Westwater et.al(2018) Psychiatry Res Cortical The cortical thickness
thickness and in the left middle frontal gyrus, right

There was no cant difference between BN and $\mathrm{HC}$ in GMV, WMV and TIV(P>0.05). There was no significant difference between BN and $\mathrm{HC}$ in TIV(P>0.05). There were no significant differences between patients and controls in total GMV, WMV or $\operatorname{TIV}(\mathrm{P}>0.05)$. 
Neuroimaging.2018;271:118-

125 structural connectivity superior frontal gyrus

and bilateral

orbitofrontal cortex(OFC) and

temporoparietal regions

negtively related to the

severity of $\mathrm{BN}$

symptoms. The structural connectivity

in the left OFC and

middle temporal cortex

negtively related to the

severity of symptoms,

while in the right

superior parietal lobule positively related to the severity of symptoms.

Table 2 Demographic variables, clinical characteristics and whole brain tissue volumes of the subjects.

Characteristic

$$
\begin{gathered}
\mathrm{BN} \\
(\mathrm{N}=34) \\
\text { Mean } \pm \text { SD }
\end{gathered}
$$

\section{$\mathrm{HC}$} $(\mathrm{N}=34)$ Mean \pm SD
$\mathrm{P}$

$22.26 \pm 2.53 \quad$ NS

Age

$22.85 \pm 3.89$

$20.46 \pm 2.80$

$20.52 \pm 1.52$

$2.92 \pm 2.35$

$15.53 \pm 2.43$

$16.09 \pm 2.45$

$33.24 \pm 3.56$

$25.76 \pm 5.47$

RSES global score ${ }^{\mathrm{a}}$

Whole brain tissue volumes (VBM)

Grey matter volume(ml) $660.56 \pm 34.17$

$662.97 \pm 37.11$

NS

White matter volume(ml)

$525.68 \pm 39.46$

$526.02 \pm 48.38$

NS

CSF volume (ml)

$227.69 \pm 22.82$

$212.68 \pm 24.09$

$<0.05$

Total intracranial volume(ml)

$1413.94 \pm 78.41$

$1401.67 \pm 91.04$

NS

NS=not significant;BMI=body mass index;

$\mathrm{CSES}=$ the Core Self-Evaluation Scale;RSES=the Rosenberg Self-Esteem Scale

$\mathrm{a}_{\text {at }}$ the time of scaning 
Table 3 Regional GMV changes in patients with BN compared to healthy controls

\begin{tabular}{|c|c|c|c|c|c|c|}
\hline Regions & Hemisphere & $\begin{array}{l}\text { Cluster } \\
\text { size } \\
\text { (voxels) }\end{array}$ & $\begin{array}{l}\text { Number of voxels within the anatomical } \\
\text { region }\end{array}$ & $\begin{array}{c}\text { P-value } \\
\text { (FWE cluster- } \\
\text { level) }\end{array}$ & $\begin{array}{l}\text { Peak t } \\
\text { values }\end{array}$ & $\begin{array}{c}\text { MNI } \\
\text { coordinates } \\
(x, y, z)\end{array}$ \\
\hline
\end{tabular}

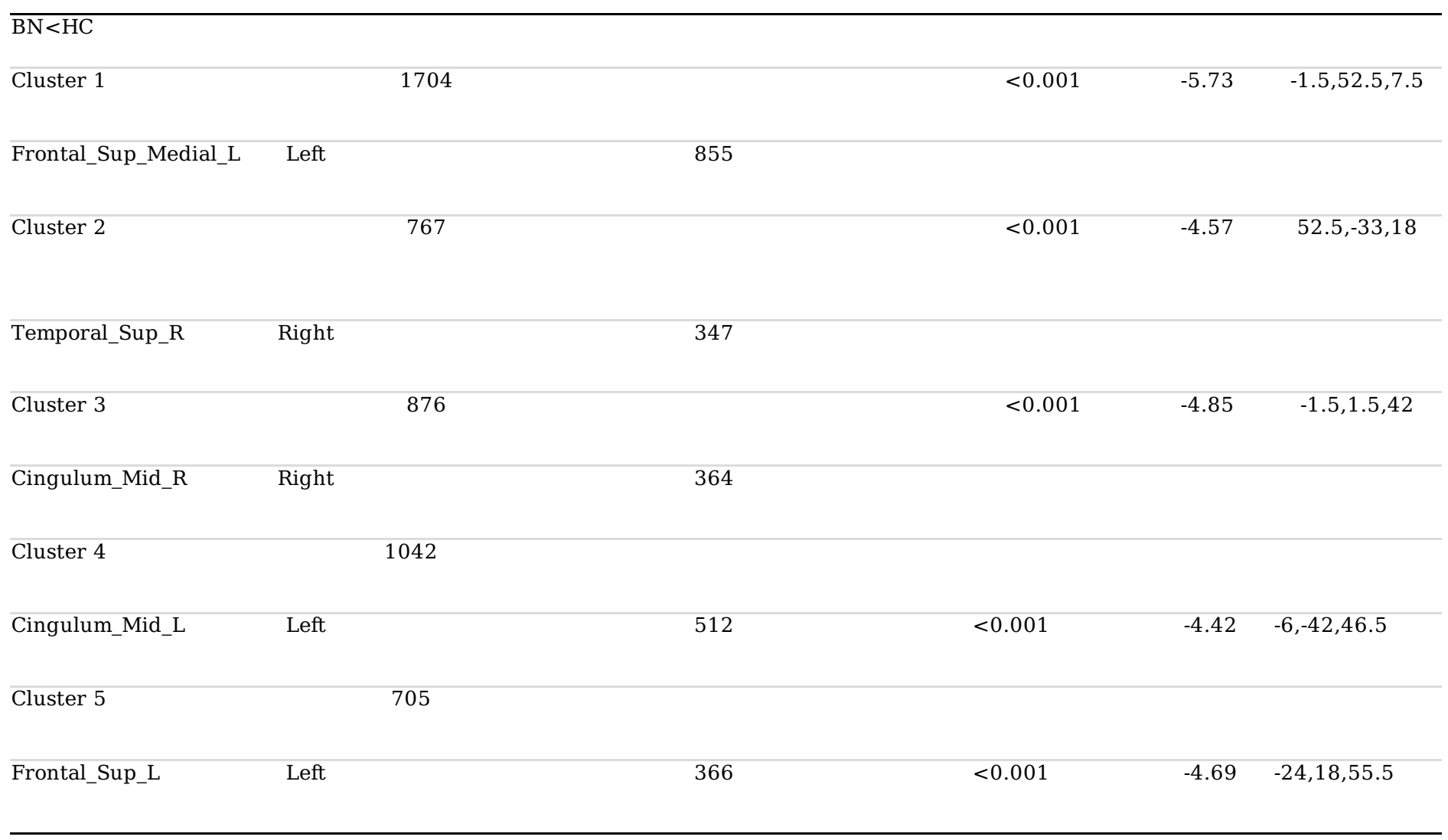

\section{Figures}



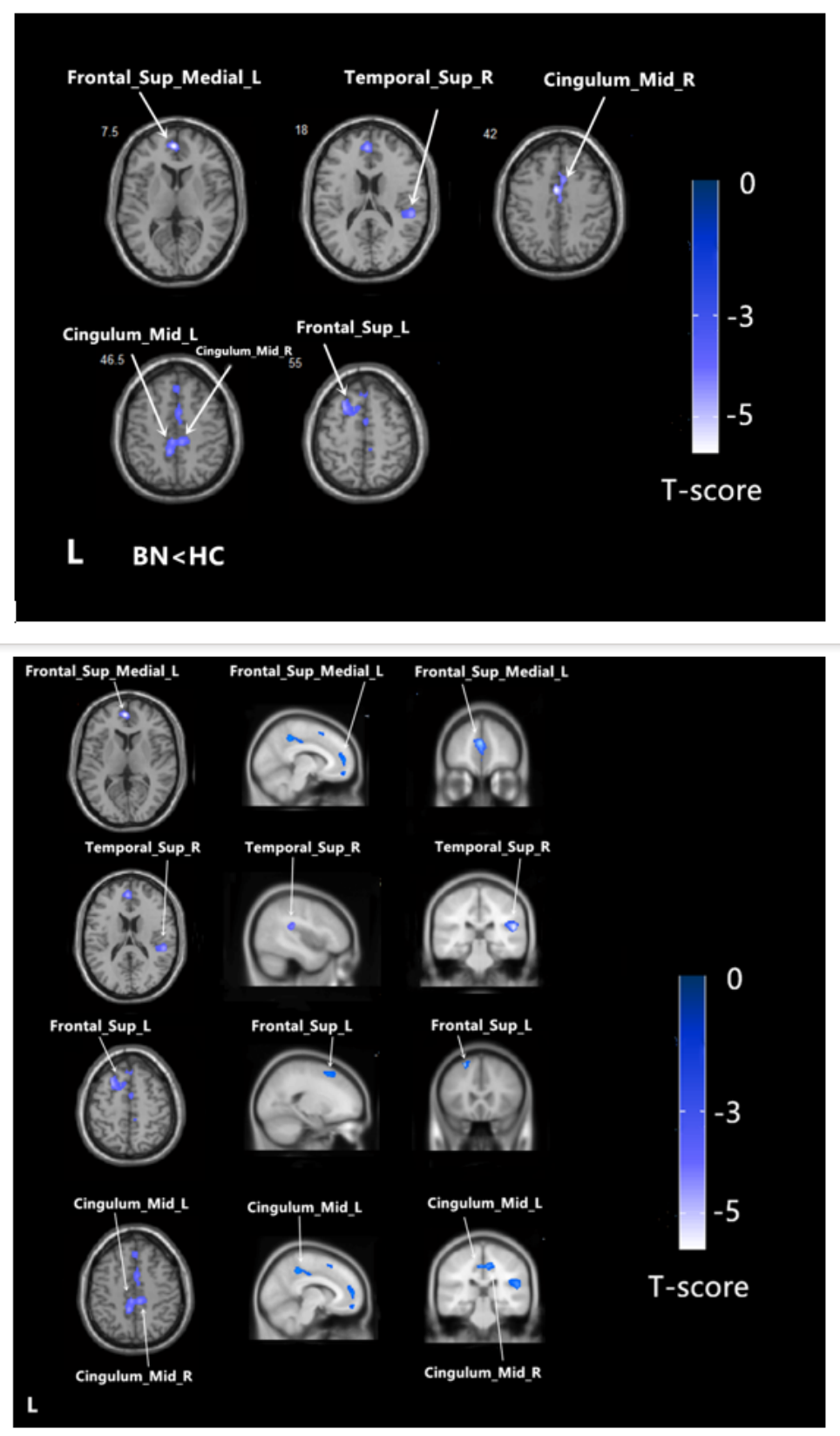

\section{Figure 1}

Regional grey matter volume alterations in $\mathrm{BN}(\mathrm{n}=34)$ relative to $\mathrm{HC}(\mathrm{n}=40)$. The statistical brain maps showed regional GMV decrease in BN, including the SFGmed.L, the STG.R, DCG.L, DCG.R and SFGdor.L ( $P<0.001$,corrected at cluster level). The color bar represents the t-scores. 

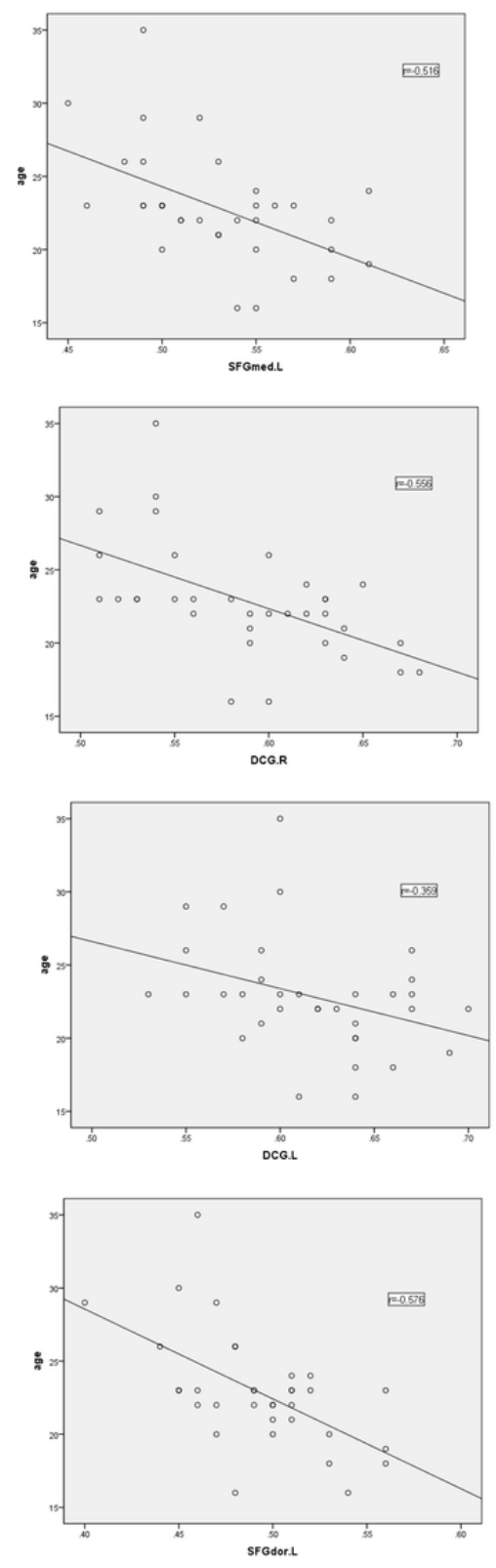

Figure 2

Correlation of regional grey matter volume with age in $\mathrm{BN}$ patients.

\section{Supplementary Files}

This is a list of supplementary files associated with this preprint. Click to download.

- Supplementmaterial.docx 